\title{
Presence of multiple peripheral circadian oscillators in the tissues controlling voiding function in mice
}

\author{
Jong-Yun Noh ${ }^{1,5}$, Dong-Hee Han ${ }^{1,5}$, Mi-Hee Kim ${ }^{1}$, Il-Gyu Ko ${ }^{2}$, Sung-Eun Kim ${ }^{2}$, Noheon Park ${ }^{3}$, \\ Han Kyoung Choe ${ }^{3}$, Khae-Hawn Kim ${ }^{4}$, Kyungjin Kim ${ }^{3}$, Chang-Ju Kim ${ }^{2}$ and Sehyung Cho ${ }^{1,2}$
}

Circadian clocks are the endogenous oscillators that harmonize a variety of physiological processes within the body. Although many urinary functions exhibit clear daily or circadian variation in diurnal humans and nocturnal rodents, the precise mechanisms of these variations are as yet unclear. In the present study, we demonstrate that Per2 promoter activity clearly oscillates in neonate and adult bladders cultured ex vivo from Per2::Luc knock-in mice. In subsequent experiments, we show that multiple local oscillators are operating in all the bladder tissues (detrusor, sphincter and urothelim) and the lumbar spinal cord (L4-5) but not in the pontine micturition center or the ventrolateral periaqueductal gray of the brain. Accordingly, the water intake and urine volume exhibited daily and circadian variations in young adult wild-type mice but not in Per $1^{-/-}$Per2 ${ }^{-/-}$ mice, suggesting a functional clock-dependent nature of the micturition rhythm. Particularly in PDK mice, the water intake and urinary excretion displayed an arrhythmic pattern under constant darkness, and the amount of water consumed and excreted significantly increased compared with those of WT mice. These results suggest that local circadian clocks reside in three types of bladder tissue and the lumbar spinal cord and may have important roles in the circadian control of micturition function. Experimental \& Molecular Medicine (2014) 46, e81; doi:10.1038/emm.2013.153; published online 7 March 2014

Keywords: bladder; circadian clock; lumbar spinal cord; peripheral oscillator; voiding; water intake

\section{INTRODUCTION}

Organisms on this rotating planet have adaptations for periodic changes in environmental conditions, such as environmental illumination, temperature, humidity, and food and predator availability. Actually, almost every life form on Earth displays obvious daily or circadian variations in biochemistry, physiology, pathology and behavior. ${ }^{1-5}$ In mammals, the suprachiasmatic nucleus of the hypothalamus acts as a master clock and orchestrates the peripheral clocks in various tissues and organs. Thus, virtually all organisms on Earth have successfully developed endogenous mechanisms that harmonize a variety of behavioral and physiological processes according to the time of day. ${ }^{2,4,5}$ With an effective circadian timing system, most physiological functions in mammals are tightly locked to their activity and rest phases to ensure optimal performance.

In accordance, distinct daily variations in urinary function and behavior are obvious in diurnal humans and nocturnal rodents. $^{6-8}$ Intuitively, most urine production and voiding should occur during the active phase, whereas better rest and sleep require increased storage of urine in the bladder and reduced urination frequency. Disruptions of proper voiding rhythms are predicted to negatively affect the organism's wellbeing. For example, nocturia, abnormal waking during the night one or more times to void, ${ }^{9-11}$ is known to decrease quality of life and negatively affects morbidity and mortality, especially in the elderly. ${ }^{12-15}$ Possible causes of nocturia have been attributed to bladder storage problems, diurnal polyuria and nocturnal polyuria, ${ }^{10}$ but the presence and functionality of local peripheral clocks in the regions controlling voiding functions have not yet been fully established. Recently, Herrera and Meredith (2010) observed day vs night differences in bladder capacity and voiding frequency in rats, raising the possibility that bladder function can be a direct target of circadian regulation. ${ }^{16}$ As nocturia is one of the most common urinary storage symptoms in most developed and

${ }^{1}$ Department of Neuroscience \& Neurodegeneration Control Research Center, Kyung Hee University, Seoul, Korea; ${ }^{2}$ Department of Physiology, Kyung Hee University School of Medicine, Seoul, Korea; ${ }^{3}$ Department of Biological Sciences, Seoul National University, Seoul, Korea and ${ }^{4}$ Department of Urology, Gachon University Gil Hospital, Gachon University of Medicine and Science, Incheon, Korea

${ }^{5}$ These authors contributed equally to this work.

Correspondence: Professor S Cho, Department of Neuroscience \& Neurodegeneration Control Research Center, Kyung Hee University, Seoul 130-701, Republic of Korea. 
developing countries, ${ }^{17,18}$ a clearer understanding of voiding function and dysfunction is necessary. The possible existence of local peripheral clocks in the tissues controlling micturition function has yet to be determined.

In the present study, we demonstrate operating circadian oscillations in the lumbar spinal cord as well as in three distinct tissues of the bladder. Moreover, we provide evidence that circadian variations in micturition function are dependent on a functional molecular clock.

\section{MATERIALS AND METHODS}

Animals, entrainment and circadian sampling

All animal experiments were approved by and performed under the guidelines of Kyung Hee University Institutional Animal Care and Use Committee. The Per1 ${ }^{-/}$Per2 ${ }^{-/-}$(PDK) ${ }^{19}$ and Per2::Luc knock-in ${ }^{20}$ mice were described previously. All mice were raised in a light-proof Clean Animal Rack cabinet (Shin Biotech, Seoul, Korea) with constant air ventilation, and these cabinets were maintained in a temperature-controlled room $\left(23 \pm 1{ }^{\circ} \mathrm{C}\right)$. The light intensity during the light phase was kept at 350-450 lux throughout the area. Young adult WT, PDK and Per2::Luc knock-in mice were entrained to a 12-h light/12-h dark (LD, light/dark 12:12) photoperiodic cycle with lights on at 0800 hours. After 1 or 2 weeks of entrainment, the mice were kept in constant darkness (DD). On the second day after the lights were turned off, the animals were sacrificed by injection of chloral hydrate $\left(360 \mathrm{mg} \mathrm{kg}^{-1}\right.$, intraperitoneal) at the indicated circadian time.

\section{Real-time monitoring of Per2 promoter activity from bladder and spinal cord}

Adult Per2::Luc knock-in mice were anesthetized with chloral hydrate (360 $\mathrm{mg} \mathrm{kg}^{-1}$, intraperitoneal). Whole bladder samples, dissected bladder tissues or spinal cord fragments (lumbar 4-5) were used for real-time monitoring of Per2 promoter activity, depending on the experiment. For the whole bladder samples, the bladder outlet was tied using thread, the remaining urine was removed using a syringe, and the bladder was filled with MEM (Gibco, Carlsbad, CA, USA) supplemented with $25 \%$ of Gey's balanced salt solution, $25 \%$ fetal bovine serum, 100 units $\mathrm{ml}^{-1}$ penicillin, $100 \mu \mathrm{g} \mathrm{ml}^{-1}$ streptomycin, $36 \mathrm{~mm}$ glucose and $0.1 \mathrm{~mm}$ luciferin (Promega, Madison, WI, USA). The whole bladder samples were immobilized in a $35-\mathrm{mm}$ culture dish (Corning, Tewksbury, MA, USA) using cell strainers (SPL, Seoul, Korea), and the bioluminescence was continuously recorded using a KRONOS apparatus (ATTO, Tokyo, Japan). To monitor neonate bladders, bladders were obtained from newborn mice, and tissues were sliced with a blade. The tissue slices were immobilized in a culture insert and incubated for 1 day after a medium change. Then, the tissue slices were synchronized by a dexamethasone shock for $3 \mathrm{~h}$. These tissues were monitored using the same procedure as for the adult bladder tissues. For the bladder tissue samples and the spinal cord, samples were obtained under a dissecting microscope and cultured separately on Millicell culture membrane inserts (Millipore, Billerica, MA, USA) with $1 \mathrm{ml}$ of MEM, supplemented with $25 \%$ of Gey's balanced salt solution, $25 \%$ fetal bovine serum, 100 units $\mathrm{ml}^{-1}$ penicillin, $100 \mu \mathrm{g} \mathrm{ml}^{-1}$ streptomycin and $36 \mathrm{~mm}$ glucose. The samples were then transferred to $35-\mathrm{mm}$ culture dishes with recording medium. The bioluminescence was continuously recorded in the same manner as for the whole bladder.
RNA isolation, reverse transcription and real-time PCR Total RNA was extracted from the bladder (urothelium, detrusor smooth muscle or sphincter smooth muscle), spinal cord (lumbar $4-5)$, pontine micturition center (PMC) and ventrolateral periaqueductal gray (vlPAG) fragments using the single-step acid guanidinium thiocyanate-phenol-chloroform extraction method as described previously. ${ }^{21}$ The RNA concentration was determined using an ND-1000 (Nanodrop Technologies, Wilmington, DE, USA). One $\mu \mathrm{g}$ of total RNA and $200 \mathrm{ng}$ of random hexamers (Takara, Shiga, Japan, 3801) in a total volume of $11 \mu \mathrm{l}$ were incubated for $5 \mathrm{~min}$ at $65^{\circ} \mathrm{C}$ and chilled on ice. Then, $4 \mu \mathrm{l}$ of $5 \mathrm{X}$ Reverse transcriptase buffer, $4 \mu \mathrm{l}$ of $2.5 \mathrm{~mm}$ each of dNTPs, $0.5 \mu \mathrm{l}$ of RNase inhibitor (40 units, Takara, 2310A) and $0.5 \mu \mathrm{l}$ of Reverse transcriptase M-MLV (200 units, Takara, 2640 A) were added, and the reaction mixture was incubated for $1 \mathrm{~h}$ at $37^{\circ} \mathrm{C}$ and for $10 \mathrm{~min}$ at $70^{\circ} \mathrm{C}$. The procedure for real-time RT-PCR using LightCycler has been described previously. ${ }^{22}$ The standards were prepared by pooling fivefold diluted cDNA samples in $1 \mathrm{~mm}$ Tris. The 5 -fold diluted cDNA samples were further diluted 15-fold to prepare templates for real-time RT-PCR. Real-time RT-PCR was performed on a LightCycler Version 1.5 (Roche, Indianapolis, IN, USA) using 2X SYBR Premix EX Taq (Takara, RR041A). The expression levels of Gapdh were used for normalization. The following primer sequences were used for real-time RT-PCR: Bmall up (upregulated), 5'-GGCC ATCAGTAAAGGTGGAA-3'; Bmall dn (downregulated), 5'-GGTGG CCAGCTTTTCAAATA- $3^{\prime}$; Rev-erb $\alpha$ up, $5^{\prime}$-AAGACATGACGACCTG GAC-3'; Rev-erb $\alpha$ dn, 5'-GAGTCAGGGACTGGAAGCTG-3'; Per1 up, $5^{\prime}$-GTGTCGTGATTAAATTAGTCAG-3'; Per1 dn, 5'-ACCACTCATG TCGTCTGGGCC-3'; Cry1 up, 5'-CGAATGAATGCAAACTCCCT-3'; Cry $1 \mathrm{dn}, 5^{\prime}$-AAAAATTCACGCCACAGGAG-3'; Gapdh up, 5'-AAC TTTGGCATTGTGGAAGG-3'; and Gapdh dn, 5'-ACACATTGGGGG TAGGAACA- $3^{\prime}$.

\section{Measurement of water intake and urine excretion}

To determine the water intake and urine excretion, WT and PDK mice (8-10 weeks) were housed individually in metabolic cages (Jeungdo, Seoul, Korea). These animals were entrained to a 12-h light/12-h dark (LD 12:12) photoperiodic cycle with lights on at 0800 hours for a week. On the 8th day, the water intake and urine excretion were measured at $2 \mathrm{~h}$ intervals throughout a day. Then, the lights were turned off on the 9th day. On the second day after the lights were turned off, the water intake and the urine excretion were determined every $2 \mathrm{~h}$ in the DD condition throughout a circadian cycle.

\section{Statistical analysis}

Water intake and urine excretion were statistically analyzed by Student's $t$-test for comparison between the two groups. Statistical significance was set at $P<0.05$.

\section{RESULTS}

Rhythmic oscillation of Per2 promoter activity in the whole bladders of neonate and adult mice

To verify the existence of an autonomous circadian clock in the isolated organotypic whole bladder, we utilized Per2::Luc knock-in mice ${ }^{20,23}$ because these animals allow us to detect the oscillation in the transcription of a canonical clock gene, Per2, in whole organs or tissues quite easily. As shown in Figures $1 \mathrm{a}$ and $\mathrm{b}$, organotypic ex vivo cultures of the whole bladder from adult as well as neonate mice displayed rhythmic oscillations of the Per2 promoter activity with a free-running 

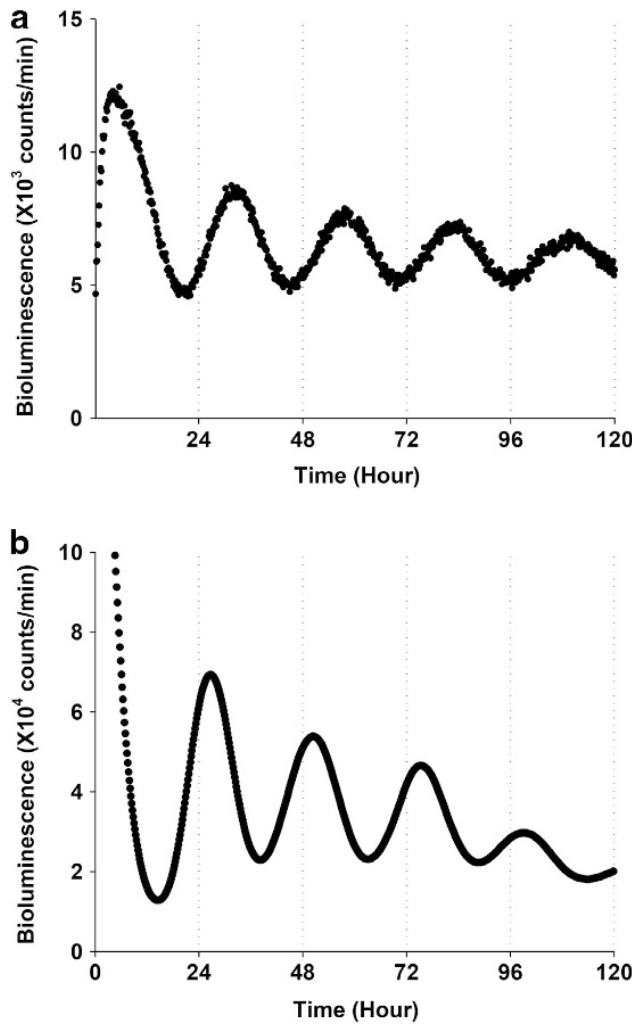

Figure 1 Representative recordings of the bioluminescence from the whole bladders of Per2::Luc mice. Whole bladders from neonate (a) and young adult (b) Per2::Luc mice were analyzed for Per2 promoter-driven luciferase activity. (a) Adult Per2::Luc knockin mice were anesthetized with chloral hydrate $\left(360 \mathrm{mg} \mathrm{kg}^{-1}\right.$, intraperitoneal), and the whole bladders were retrieved. Then, the bladder outlet was tied using thread, the remaining urine was removed using a syringe, and the bladder was filled with MEM (Gibco) supplemented with the appropriate medium. The whole bladder samples were immobilized in a 35-mm culture dish (Corning, Tewksbury, MA, USA) using cell strainers (SPL, Seoul, Korea), and the bioluminescence was continuously recorded using a KRONOS apparatus (ATTO, Tokyo, Japan). (b) To monitor neonate bladders, bladders were obtained from newborn mice, and the tissues were sliced using a blade. The tissue slices were immobilized in a culture insert and incubated for 1 day after a medium change. Then, the tissue slice were synchronized by dexamethasone shock for $3 \mathrm{~h}$. Monitoring was performed using the same method as for the adult bladder tissues. The bioluminescence was continuously recorded for more than 5 days using a KRONOS apparatus as described in Methods.

period of $\sim 24 \mathrm{~h}$. The oscillation of Per2 promoter activity in the isolated whole bladders indicates that the Per2 promoter is active in the bladder of neonate and adult mice and that the rhythmic transcription of Per2 gene is intrinsic to a bladder that is operating without any cues from other body parts.

Presence of functional peripheral clocks in three distinct tissues of the bladder

The urinary bladder can be divided into three functional parts: detrusor smooth muscle, sphincter smooth muscle, and urothelium. ${ }^{24,25}$ Once we confirmed the intrinsic oscillations of Per2 promoter activity, our next question was whether circadian clocks operate in the three distinct functional tissues of the bladder. To this end, we rapidly obtained these tissues under a dissecting microscope and continuously recorded the resulting bioluminescence for 5 days using a KRONOS apparatus. In addition, we examined the expression patterns of other clock genes (Per1, Bmall and Rev-erb $\alpha$ ) over a circadian cycle in DD by quantitative real-time RT-PCR. As shown in Figure 2, all the functional parts of the bladder exhibited distinct and persistent oscillations in Per2 bioluminescence with a period of $\sim 24 \mathrm{~h}$. The periods and acrophases were indistinguishable among the three types of tissue. Moreover, all canonical clock genes examined in this study oscillated in the wild-type mice but not in the arrhythmic Per1 ${ }^{-/-}$ Per2 ${ }^{-1-}$ (Pers double knockout; PDK) mice. The persistent oscillation of circadian clock genes in separated bladder tissues indicates the intrinsic nature of local peripheral clocks in the three functional tissues of the bladder.

\section{Functional clockwork also operates in the lumbar spinal cord of mice}

The control of voiding functions by central nervous system inputs is well known. In particular, the lumbar spinal cord has been connected to voiding and urine storage reflexes. ${ }^{25}$ Thus, we investigated whether functional circadian clockwork also exists in this part of the spinal cord, that is, lumbar 4-5 region. We first examined the Per 2 promoter activity in the isolated lumbar spinal cords from Per2::Luc mice (Figure 3; upper left), which revealed a persistent circadian oscillation in the bioluminescence. In addition, the expression of other clock genes was analyzed by real-time RT-PCR, which demonstrated that Per1, Bmall and Rev-erb $\alpha$ oscillated in the lumbar spinal cord in the WT mice in the absence of an exogenous LD cycle (Figure 3); however, the amplitudes were smaller than those of the bladder tissues (compare with Figure 2). As expected, the PDK mice did not exhibit any significant oscillation. These results indicate the presence of functional circadian clockwork in the lumbar spinal cord, which operates intrinsically without cues from other body parts.

No oscillations of clock genes in the PMC and vlPAG

Higher brain regions, such as the PMC and vlPAG, are also involved in the neural control of micturition function. ${ }^{24-27}$ Thus, we investigated whether any functional circadian clockworks exist in these regions of the brain (Figure 4). Although the expression of clock genes was evident in these regions, no clear oscillation was detected by quantitative realtime RT-PCR. Moreover, the Rev-erb $\alpha$ expression levels of the WT mice were similar to those of PDK mice, indicating the absence of a functional peripheral clock in the PMC and vlPAG.

Daily patterns of water intake and urine volume in WT and PDK mice: effect of an exogenous LD cycle

After establishing the existence of peripheral circadian clockworks in some of the voiding-related tissues, our next focus 

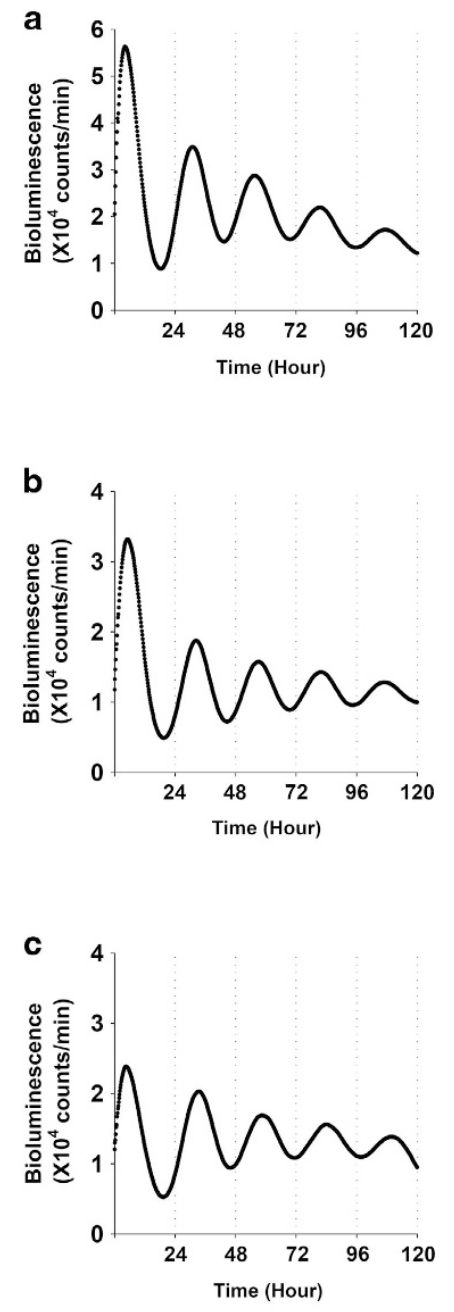
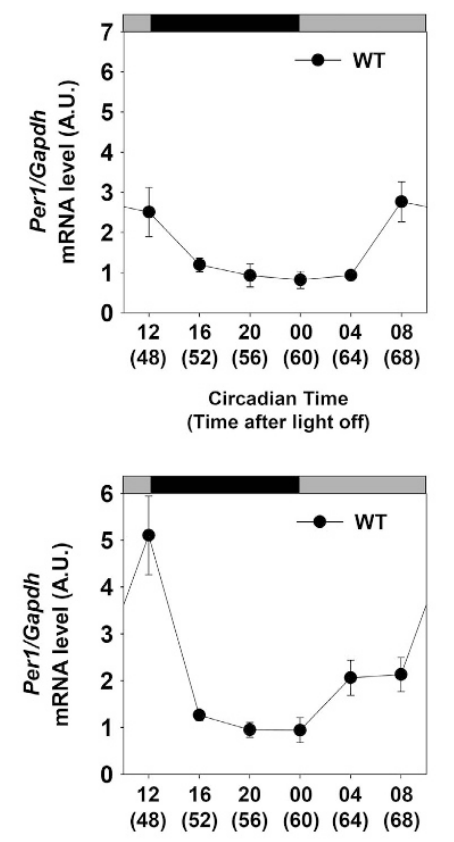

$$
\begin{gathered}
\text { Circadian Time } \\
\text { (Time after light off) }
\end{gathered}
$$

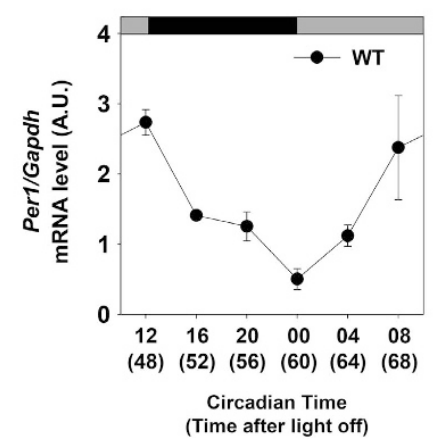

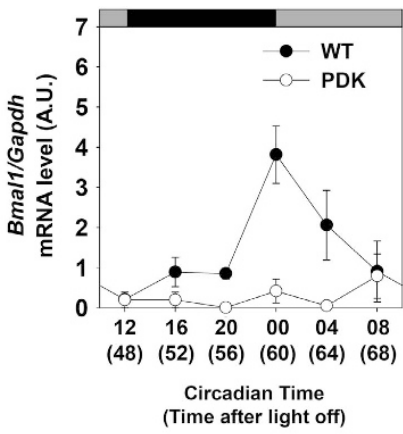
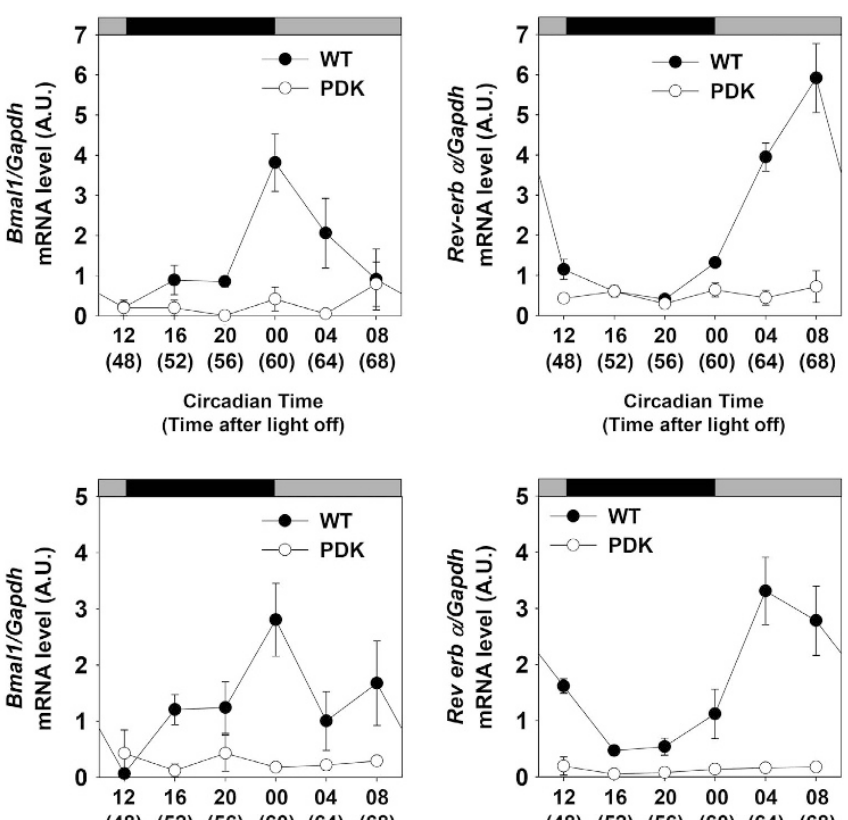

(48) (52) (56) (60) (64) (68)

Circadian Time (Time after light off)

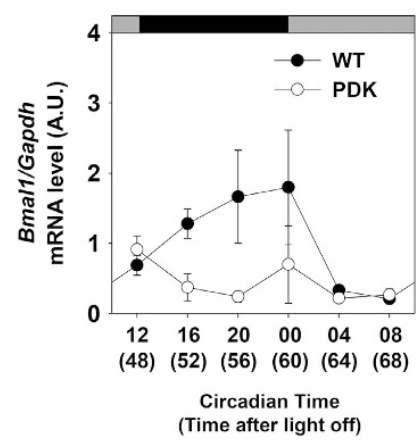

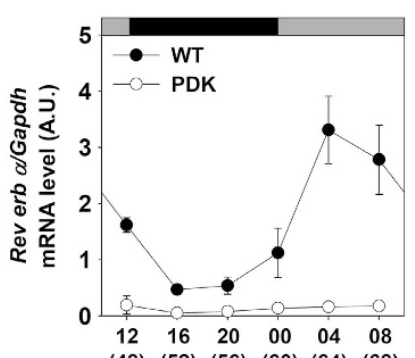

(48) (52) (56) (60) (64) (68)

Circadian Time (Time after light off)

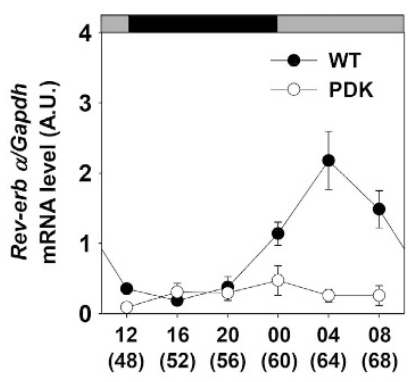

Circadian Time

(Time after light off)

Figure 2 Existence of functional circadian clockworks in three distinct tissues of the bladder. (Far left panels of a-c) Detrusor smooth muscle (a), sphincter smooth muscle (b), and uroepithelial layer (c) of adult Per2::Luc mouse bladders were obtained under a dissecting microscope and analyzed for Per2 promoter-driven luciferase activity. The bioluminescence was continuously recorded for more than 5 days using a KRONOS apparatus as described in Methods. Representative recordings of bioluminescence are shown here. (Next three panels of a-c) Expression of clock genes in three bladder tissues of WT and PDK mice. WT and PDK mice were entrained to a 12L:12D photoperiodic cycle for a week and then kept in constant darkness (DD). Two days after the lights were turned off, the mice were sacrificed by cervical dislocation at the indicated circadian time, and the detrusor smooth muscle (a), sphincter smooth muscle (b) and uroepithelial layer (c) were quickly obtained under a dissecting microscope. Real-time RT-PCR was performed to determine the mRNA levels of Per1, Bmal1 and Rev-erba. All mRNA levels were normalized to the Gapdh mRNA level and expressed as the mean \pm s.e.m. $(n=4)$. L, light; D, dark; PDK, double knockout of Per1 and Per2 genes, WT, wild type.

was determining their functional significance. Thus, we examined water intake and voiding volume not only in WT mice but also in arrhythmic PDK mice. To establish daily patterns of water intake and urine excretion, males of both genotypes were individually housed in metabolic cages and entrained to a 12L:12D photoperiodic cycle for a week. Then, their water intake and urine volume were simultaneously determined every $2 \mathrm{~h}$ throughout the eighth day (Figure 5a). In the presence of an LD cycle, the WT male mice exhibited daily variations in water intake and urine volume with an increased amount of water consumed and excreted during the active/ dark phase. Furthermore, daily variations in water intake and urine volume were evident in the PDK male mice, although the day vs night difference in urine volume was statistically insignificant. This observed day vs night difference in PDK mice is consistent with the seemingly rhythmic locomotor activity observed in the presence of an exogenous LD cycle. ${ }^{28,29}$ Interestingly, the PDK mice consumed more water than did the WT mice at the time of the light-dark transition and showed a subsequent increase in urine volume $2-4 \mathrm{~h}$ after the increased water consumption, suggesting that water intake and urine volume in PDK mice are largely affected by the presence 

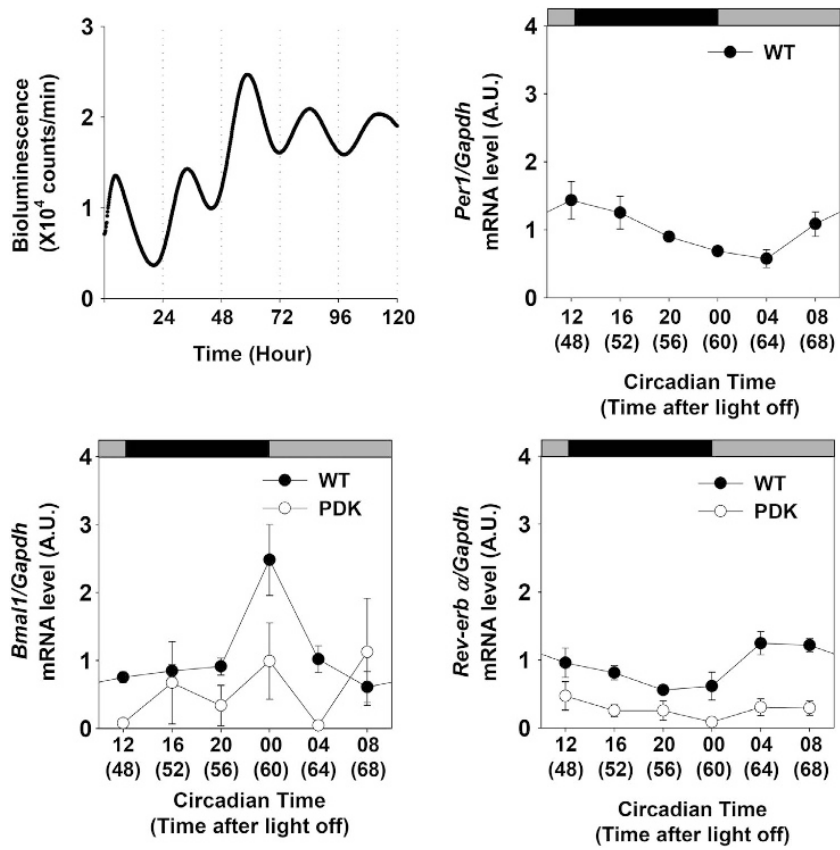

Figure 3 A functional circadian oscillator in the lumbar spinal cord. (Upper left) A representative recording of the bioluminescence from the lumbar spinal cord of Per2::Luc mice. The bioluminescence was measured from cultures of spinal cord fragments (L4-5) for more than 5 days, as in Figure 1. (Next three panels) WT and PDK mice were entrained to a 12L:12D photoperiodic cycle for a week and then kept in constant darkness (DD). Two days after the lights were turned off, the mice were sacrificed by cervical dislocation at the indicated circadian time, and the spinal cord (lumbar 4-5 region) was quickly obtained. Real-time RT-PCR was performed to determine mRNA levels of Per1, Bmal1 and Rev-erba. All mRNA levels were normalized to the Gapdh mRNA level and expressed as the mean \pm s.e.m. $(n=4)$. L, light; D, dark; PDK, double knockout of Per1 and Per2 genes, WT, wild type.

of exogenous light. Moreover, the 24-h water intake and urine volume of the PDK mice were higher than those of the WT in most cases, suggesting polydipsia and polyuria in these mutant animals.

Circadian patterns of water intake and urine volume in WT and PDK mice: intrinsic nature of water consumption and excretion rhythms

To determine whether the apparent rhythmic pattern observed in the PDK mice under the LD condition is due to the masking effect of exogenous light, the lights were turned off on the 9th day. On the second day after the lights were turned off, the water intake and the urine volume were simultaneously determined every $2 \mathrm{~h}$ throughout a circadian cycle under the DD condition (Figure 5b). Even in the DD, the WT mice still exhibited significant variations in water intake and urine volume, showing a subjective night vs subjective day difference. In contrast, the PDK mice quickly lost their apparent rhythms of water intake and excretion under the same condition. The complete loss of rhythmicity in the PDK mice suggests an endogenous clock-dependent nature of water consumption and urinary excretion in WT mice. The time at which the minimal volumes of urine were collected is particularly notable. Indeed, the urine volumes reached the minimum $3-4 \mathrm{~h}$ before the light-dark transition in the WT mice (Figure 5a), and this minimum was maintained in the WT mice even in the absence of an exogenous lighting cue (Figure 5b). Thus, we can conclude that the urine volume in WT mice is dependent on the functional clock but not on the exogenous light or the locomotor activity level.

\section{DISCUSSION}

Extensive studies have demonstrated diurnal or circadian variations in urine volume, electrolyte excretion, uroflow, voiding frequency, volume per void, and urine and osmole output rates. ${ }^{7,30-34}$ Moreover, arginine vasopressin synthesized in the magnocellular neurons of the hypothalamus displays distinct diurnal or circadian variations and is implicated in many urological problems. ${ }^{35-37}$ However, the presence of circadian oscillators in the local regions that control voiding function has so far been largely unnoticed. In the present work, we demonstrated that local circadian clockworks operate intrinsically in the bladder and lumbar spinal cord, the local regions that control voiding function in mice, even in the absence of extrinsic time cues. We also provided evidence that water intake and urinary excretion are controlled in a functional clock-dependent manner, with a possible indication of polydipsia and polyuria in clock-defective PDK mice.

The present work clearly demonstrated persistent oscillation of clock genes not only in the whole bladder but also in three isolated bladder tissues (detrusor, sphincter and urothelim) and lumbar spinal cord fragments (L4-5); the oscillation periods of $\sim 24 \mathrm{~h}$ were similar. However, we failed to detect any significant oscillation in circadian genes in the PMC and vlPAG, the higher brain regions that control micturition function; therefore, the clock ticks only in some but not all micturition function-related tissues. Moreover, the amplitudes of the circadian oscillation were much higher in the bladder tissues than in the lumbar spinal cord, suggesting that the clock ticks more robustly in peripheral tissues. Although the oscillation of Per2 transcription in the bladder slice cultures has very recently been reported, ${ }^{38}$ this work is the first to demonstrate the presence of multiple peripheral oscillators in micturition function-related tissues. Detrusor smooth muscle, sphincter smooth muscle, urothelim and lumbar spinal cord have distinct roles in the control of micturition and can be separately targeted by various drugs; ${ }^{24,25,27,39}$ therefore, the roles of these peripheral oscillators must be resolved in future work.

In this study, the functional clock-defective PDK mice displayed daily variations in water intake and urine volume in the presence of an exogenous LD cycle (Figure 5a), whereas this apparent rhythmicity disappeared just 2 days after the mice were kept in DD (Figure 5b). Thus, the seemingly normal 


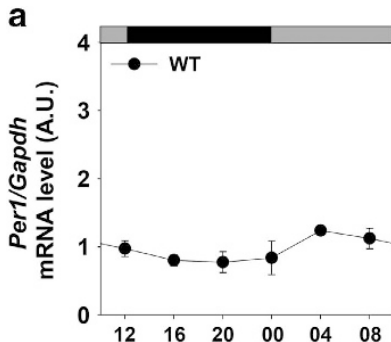

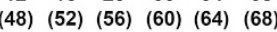

Circadian Time (Time after light off)

b

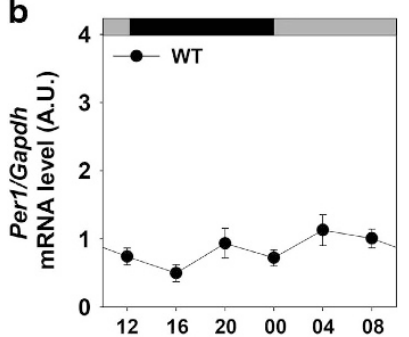

(48) (52) (56) (60) (64) (68)

Circadian Time

(Time after light off)

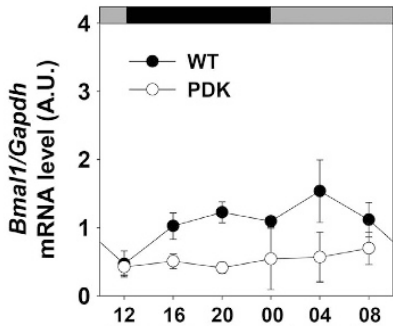

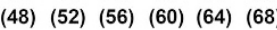

Circadian Time (Time after light off)

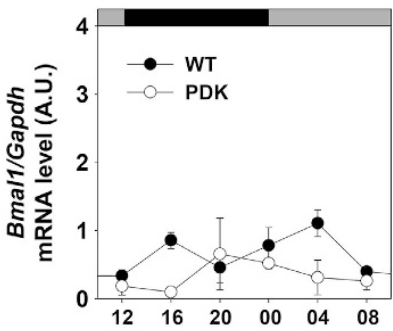

(48) (52) (56) (60) (64) (68)

Circadian Time (Time after light off)

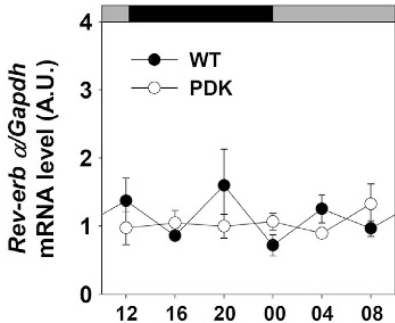

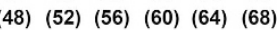

Circadian Time (Time after light off)

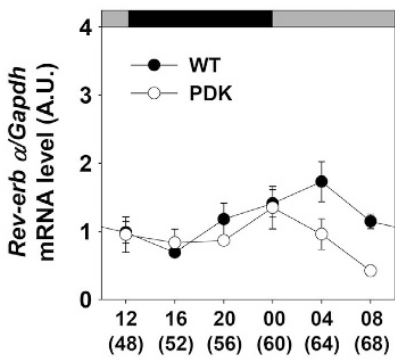

Circadian Time (Time after light off)

Figure 4 No evident rhythms in the expression clock genes in the PMC and vIPAG. WT and PDK mice were entrained to a 12L:12D photoperiodic cycle for a week and then kept constant darkness (DD). Two days after the lights were turned off, mice were sacrificed by cervical dislocation at the indicated circadian time. The pontine micturition center (PMC, a) and ventrolateral periaqueductal gray (vIPAG, b) were quickly obtained under a dissecting microscope. Real-time RT-PCR was performed to determine the mRNA levels of Per1, Bmal1, and Rev-erbo. All mRNA levels were normalized to the Gapdh mRNA level and expressed as the mean \pm s.e.m. ( $n=4)$. L, light; D, dark; PDK, double knockout of Per1 and Per2 genes, WT, wild type.
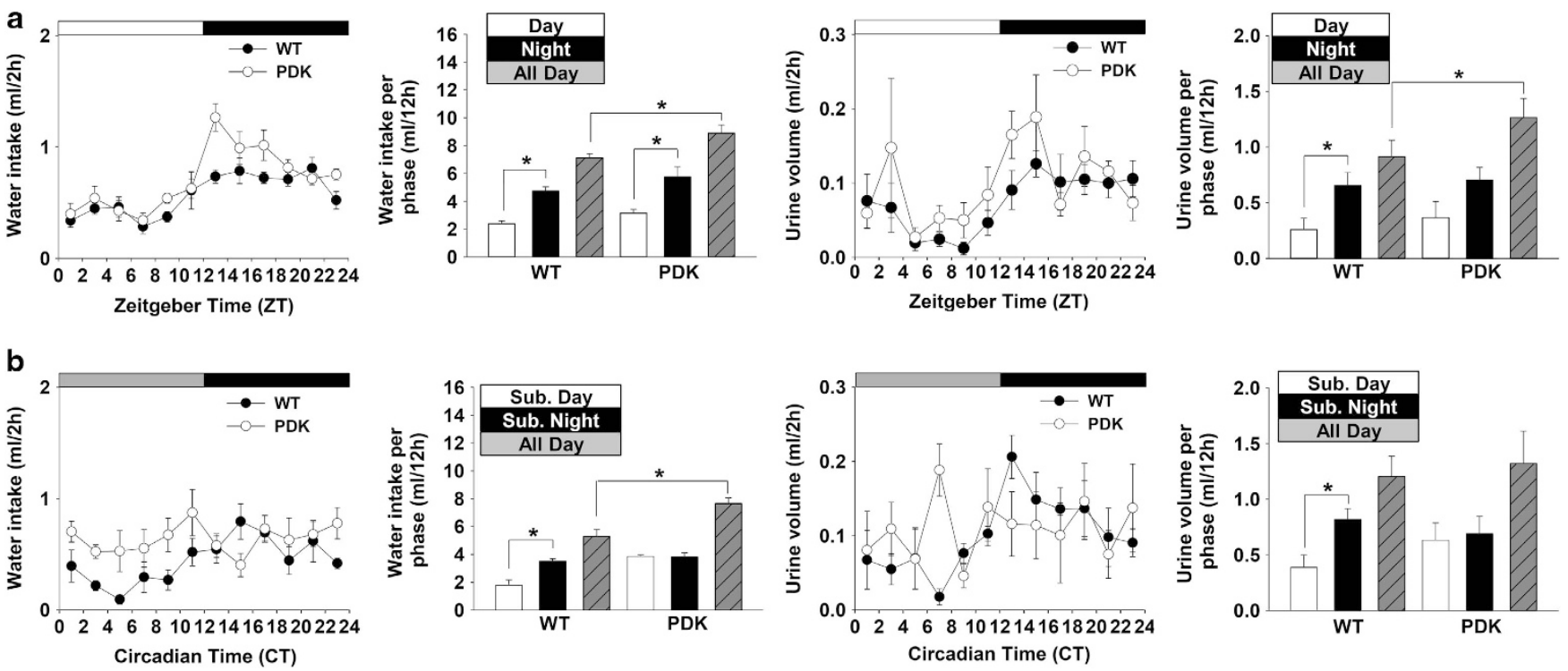

Figure 5 Daily and circadian patterns of water intake and urine excretion in WT and PDK mice. (a) Daily patterns of water intake and urine excretion. WT and PDK mice were housed individually in metabolic cages. These animals were further entrained to a 12-h light/ 12-h dark (LD 12:12) photoperiodic cycle with lights on at 0800 hours for a week. On the 8th day, the water intake and urine excretion were determined at 2-h intervals throughout a day and are shown as line graphs. Then, the 12-h day, 12-h night and 24-h all-day amounts were pooled and are shown as bar graphs on the right side. (b) Circadian patterns of water intake and urine excretion. To determine the circadian patterns, the lights were turned off on the 9th day. Two days after the lights were turned off, the water intake and urine excretion under the DD condition were determined at 2-h intervals throughout a circadian cycle and are shown as line graphs. Then, the 12-h subjective day, 12-h subjective night and 24-h all-day amounts were pooled and are shown as bar graphs on the right side (b). ${ }^{*} P<0.05$. LD, light/dark; DD, constant darkness; PDK, double knockout of Per1 and Per2 genes, WT, wild type. 
rhythmicity of the PDK mice under the LD condition can be simply explained by the masking influences of the environmental light. In other words, when environmental light inhibits locomotor activity in rodents, ${ }^{28,29}$ the light-induced changes in the activity level in PDK mice lead to alterations in water intake and subsequently changes the urine volume. In support of this notion, the PDK mice consumed the highest amount of water especially at the time of the light-dark transition (Figure 5a) when their locomotor activity also intensifies. ${ }^{29}$ The involvement of external time cues as well as the central rhythm that is conveyed to the voiding organs presumably via neural inputs in these processes cannot, however, be ruled out. Thus, the statistical significance was greater for the LD condition than for DD condition. Two-way analysis of variance showed statistical significance for LD depended on the genotype $(P<0.001)$ and ZT $(P<0.001)$ but not for their interaction $(P>0.05)$, whereas under the DD condition, statistical significance was obtained only for the genotypes $(P<0.001)$.

We also observed that the water intake and urine excretion substantially increased in the PDK mice compared with those in the WT mice, possibly indicating polydipsia and polyuria in the PDK mice. Recently, Zuber et al. (2009) reported that Clock $^{-1-}$ mice exhibit diverse phenotypes, including partial diabetes insipidus. ${ }^{40}$ Indeed, disturbances of circadian rhythms or knockouts of circadian clock genes have been linked to various pathologies, including mental illness, cancer, obesity, diabetes, cardiovascular disease and aging. ${ }^{4,41}$ As discussed previously, ${ }^{6-8}$ most urine production and voiding should occur during the active phase, whereas better rest and sleep require increased storage of urine in the bladder and reduced voiding frequency. Thus, disruption of the endogenous clockwork in PDK mice may result in urinary problems, such as polyuria. However, the exact mechanism must be clarified in future works. In this respect, a recent report by Negoro et al. (2012) showing the circadian oscillation of connexin 43 in the control of bladder capacity provides a good exemplar. ${ }^{38}$ However, more extensive studies are required to address other possibilities. In particular, mouse models carrying tissue-specific disruptions of molecular clockwork are needed to address the tissue-specific roles of local circadian oscillators in voiding function and dysfunction. Because functional circadian clockwork exists in three distinct bladder tissues and the lumbar spinal cord but not in the PMC and vlPAG, tissue-specific ablation of circadian clocks may reveal tissue-specific functions of the local peripheral clocks.

In conclusion, we demonstrated the presence of intrinsically operating molecular clockwork in all the bladder tissues and the lumbar spinal cord. The present work opens a new research avenue for studying the possible roles of peripheral clocks in voiding function and dysfunction.

\section{ACKNOWLEDGEMENTS}

This work was supported by research grants funded by the Korean government (MEST) (Nos. 2011-0000929 and 2011-0027432) through the KRF. Noh JY and Kim KH were also supported by Astellas Pharma Korea.

1 Dunlap JC. Molecular bases for circadian clocks. Cell 1999; 96: 271-290.

2 Levi F, Schibler U. Circadian rhythms: mechanisms and therapeutic implications. Annu Rev Pharmacol Toxicol 2007; 47: 593-628.

3 Hastings MH, Maywood ES, O'Neill JS. Cellular circadian pacemaking and the role of cytosolic rhythms. Curr Biol 2008; 18: R805-R815.

4 Takahashi JS, Hong HK, Ko CH, McDearmon EL. The genetics of mammalian circadian order and disorder: implications for physiology and disease. Nat Rev Genet 2008; 9: 764-775.

5 Parch CL, Green CB, Takahashi JS. Molecular architecture of the mammalian circadian clock. Trends Cell (e-pub ahead of print 31 July 2013; doi:10.1016/j.tcb.2013.07.002).

6 Cho S, Han DH, Kim DH. Circadian rhythms in voiding function and dysfunction. Int Neurourol J 2011; 15: 2-3.

7 Noh JY, Han DH, Yoon JA, Kim MH, Kim SE, Ko IG et al. Circadian rhythms in urinary functions: possible roles of circadian clocks? Int Neurourol J 2011; 15: 64-73.

8 Negoro H, Kanematsu A, Yoshimura K, Ogawa O. Chronobiology of micturition: putative role of the circadian clock. J Urol 2013; 190: 843-849.

9 van Kerrebroeck P, Abrams P, Chaikin D, Donovan J, Fonda D, Jackson S et al. The standardisation of terminology in nocturia: report from the standardisation sub-committee of the International Continence Society. Neurourol Urodyn 2002; 21: 179-183.

10 Brunner A, Riss P. Nocturia in women. Maturitas 2011; 70: 16-21.

11 Breyer BN, Shindel AW, Erickson SB, Blaschko SD, Steers WD, Rosen RC. The association of depression, anxiety and nocturia: a systematic review. J Urol 2013; 190: 953-957.

12 Asplund R. Mortality in the elderly in relation to nocturnal micturition. BJU Int 1999; 84: 297-301.

13 Ali A, Snape J. Nocturia in older people: a review of causes, consequences, assessment and management. Int J Clin Pract 2004; 58: 366-373.

14 Kujubu DA, Aboseif SR. An overview of nocturia and the syndrome of nocturnal polyuria in the elderly. Nat Clin Pract Nephrol 2008; 4: 426-435.

15 Nakagawa H, Niu K, Hozawa A, Ikeda Y, Kaiho Y, Ohmori-Matsuda K et al. Impact of nocturia on bone fracture and mortality in older individuals: a Japanese longitudinal cohort study. J Urol 2010; 184: 1413-1418.

16 Herrera GM, Meredith AL. Diurnal variation in urodynamics of rat. PLOS One 2010; 5: e12298.

17 Irwin DE, Milsom I, Hunskaar S, Reilly K, Kopp Z, Herschorn S et al. Population-based survey of urinary incontinence, overactive bladder, and other lower urinary tract symptoms in five countries: results of the EPIC study. Eur Urol 2006; 50: 1306-1314.

18 Choo MS, Ku JH, Park CH, Lee YS, Lee KS, Lee JG et al. Prevalence of nocturia in a Korean population aged 40 to 89 years. Neurourol Urodyn 2008; 27: 60-64.

19 Bae K, Jin X, Maywood ES, Hastings MH, Reppert SM. Differential functions of mPer1, mPer2, and mPer3 in the SCN circadian clock. Neuron 2001; 30: 525-536.

20 Yoo SH, Yamazaki S, Lowrey PL, Shimomura K, Ko CH, Buhr ED et al. PERIOD2::LUCIFERASE real-time reporting of circadian dynamics reveals persistent circadian oscillations in mouse peripheral tissues. Proc Natl Acad Sci USA 2004; 101: 5339-5346.

21 Cho S, Cho H, Geum D, Kim K. Retinoic acid regulates gonadotropinreleasing hormone $(\mathrm{GnRH})$ release and gene expression in the rat hypothalamic fragments and GT1-1 neuronal cells in vitro. Mol Brain Res 1998; 54: 74-84.

22 Doi M, Cho S, Yujnovsky I, Hirayama J, Cermakian N, Cato AC et al. Light-inducible and clock-controlled expression of MAP kinase phosphatase 1 in mouse central pacemaker neurons. J Biol Rhythms 2007; 22: 127-139.

23 Cheon S, Park N, Cho S, Kim K. Glucocorticoid-mediated Period2 induction delays the phase of circadian rhythm. Nucleic Acids Res 2013; 41: 6161-6174.

24 Ford AP, Gever JR, Nunn PA, Zhong Y, Cefalu JS, Dillon MP et al. Purinoceptors as therapeutic targets for lower urinary tract dysfunction. Br J Pharmacol 2006; 147 (suppl 2), S132-S143. 
25 Fowler CJ, Griffiths D, de Groat WC. The neural control of micturition. Nat Rev Neurosci 2008; 9: 453-466.

26 Abrams P, Andersson KE. Muscarinic receptor antagonists for overactive bladder. BJU Int 2007; 100: 987-1006.

27 Yoshimura N, Miyazato M, Kitta T, Yoshikawa S. Central targets for the treatment of bladder dysfunction. Neurourol Urodyn (e-pub ahead of print 5 July 2013; doi:10.1002/nau.22455).

28 Pendergast JS, Yamazaki S. Masking responses to light in period mutant mice. Chronobiol Int 2011; 28: 657-663.

29 Park N, Cheon S, Son GH, Cho S, Kim K. Chronic circadian disturbance by a shortened light-dark cycle increases mortality. Neurobiol Aging 2012; 33: e11-e22.

30 Mills JN. Diurnal rhythm in urine flow. J Physiol 1951; 113: 528-536.

31 Minors DS, Waterhouse JM. Circadian rhythms of urinary excretion: the relationship between the amount excreted and the circadian changes. J Physiol 1982; 327: 39-51.

32 van Haarst EP, Heldeweg EA, Newling DW, Schlatmann TJ. The 24-h frequency-volume chart in adults reporting no voiding complaints: defining reference values and analyzing varialves. BJU Int 2004; 93: 1257-1261.

33 Parsons M, Tissot W, Cardozo L, Diokno A, Amundsen CL, Coats AC. Bladder diary research team. Normative bladder diary measurements: night versus day. Neurourol Urodyn 2007; 26: 465-473.

34 Van Hoeck K, Bael A, Lax H, Hirche H, van Gool JD. Circadian variation of voided volume in normal school-age children. Eur J Pediatr 2007; 166: 579-584.
35 Rittig S, Knudsen UB, Norgaard JP, Pedersen EB, Djurhuus JC. Abnormal diurnal rhythm of plasma vasopressin and urinary output in patients with enuresis. Am J Physiol 1989; 256 (4 Pt 2), F664-F671.

36 Asplund R, Aberg H. Diurnal variation in the levels of antidiuretic hormone in the elderly. J Intern Med 1991; 229: 131-134.

37 Moon DG, Jin MH, Lee JG, Kim JJ, Kim MG, Cha DR. Antidiuretic hormone in elderly male patients with severe nocturia: a circadian study. BJU Int 2004; 94: 571-575.

38 Negoro H, Kanematsu A, Doi M, Suadicani SO, Matsuo M, Imamura M et al. Involvement of urinary bladder Connexin43 and the circadian clock in coordination of diurnal micturition rhythm. Nat Commun 2012; 3: 809.

39 de Groat WC. The urothelium in overactive bladder: passive bystander or active participant? Urology 2004; 64 (6 Suppl 1), 7-11.

40 Zuber AM, Centeno G, Pradervand S, Nikolaeva S, Maquelin L, Cardinaux L et al. Molecular clock is involved in predictive circadian adjustment of renal function. Proc Natl Acad Sci USA 2009; 106: 16523-16528.

$41 \mathrm{Ko} \mathrm{CH}$, Takahashi JS. Molecular components of the mammalian circadian clock. Hum Mol Genet 2006; 2: R271-R277.

(c) (1)(2) This work is licensed under a Creative Commons Attribution-NonCommercial-ShareAlike 3.0 Unported License. To view a copy of this license, visit http:// creativecommons.org/licenses/by-nc-sa/3.0/ 